\title{
Development of quantitative MRM assays for the measurement of 3,000 proteins across 20 mouse tissues
}

\author{
S.A. Michaud ${ }^{1}$, A.L. Palmer ${ }^{1}$, N.J.T. Sinclair ${ }^{1}$, H. Pětrošováa ${ }^{1}$ I. Feldmann², \\ Y. Mohammed, ${ }^{1,3}$, A. Sickmann², C.H. Borchers ${ }^{1,4,5,6}$ \\ ${ }^{1}$ University of Victoria - Genome British Columbia Proteomics Centre, Victoria, Canada \\ ${ }^{2}$ Leibniz-Institut für Analytische Wissenschaften - e.V., Dortmund, Germany North Rhine-Westphalia \\ ${ }^{3}$ Center for Proteomics and Metabolomics, Leiden University Medical Center, Leiden, \\ Netherlands South Holland \\ ${ }^{4}$ University of Victoria, Department of Biochemistry and Microbiology, Petch Building Room 207, \\ 3800 Finnerty Rd., Victoria, BC, V8P 5C2, Canada \\ ${ }^{5}$ Gerald Bronfman Department of Oncology, Jewish General Hospital, McGill University, \\ 3755 Cote-Ste-Catherine Road, Montreal, QC H3T 1E2, Canada \\ ${ }^{6}$ Jewish General Hospital Proteomics Laboratory, McGill University, Lady Davis Institute, \\ 3755 Chemin de la Côte-Sainte-Catherine, Montréal, QC H3T 1E2, Canada \\ *e-mail: christoph@proteincentre.com
}

Key words: proteomics, protein expression mouse, mass spectrometry, assay

Detailed characterization of protein expression in mouse tissues is challenging to perform due to the lack of available tools for rapid and robust quantitation. To simplify this process, we are developing highly multiplexed panels of assays to quantify 3,000 unique proteins across 20 mouse tissues by MRM mass spectrometry. Our method requires minimal sample pre-processing and uses stable isotope-labeled standard (SIS) peptides for precise and sensitive quantitation. Assay development involves determination of the LLOQ, linear range, and assay variability for each peptide. This rigorous characterization ensures the quality of each assay. Ultimately these assays will provide the first steps towards large scale, multi-tissue quantitation, and will allow researchers to gain an improved understanding of complex biological processes and diseases. 